\title{
Simple Way To Fabricate Novel Paper-Based Valves Using Plastic Comb Binding Spines
}

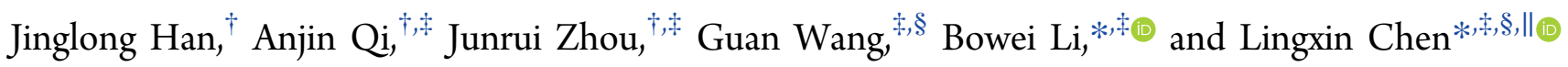 \\ ${ }^{\dagger}$ School of Environment and Materials Engineering and ${ }^{\S}$ College of Chemistry and Chemical Engineering, Yantai University, Yantai \\ 264005, China \\ "CAS Key Laboratory of Coastal Environmental Processes and Ecological Remediation, Yantai Institute of Coastal Zone Research, \\ Chinese Academy of Sciences, Yantai 264003, China \\ "College of Chemistry and Chemical Engineering, Qufu Normal University, Qufu 273165, China
}

Supporting Information

\begin{abstract}
A novel strategy for fabricating the paper-based valves on microfluidic paper-based analytical devices ( $\mu$ PADs) was described to control fluid in a user-friendly way. Initial prototypes of 3D $\mu$ PADs manipulate the spatial distribution of fluid within the device. The movable paper channel in a different layer could be achieved using the channel's connection or disconnection to realize the valve function using plastic comb binding spines (PCBS). The entire valve manipulation process was similar to a desk calendar that can be flipped over and turned back. It is notable that this kind of PCBS valve can control a fluid in a simple and easy way without the timing setting or any trigger, and this advantage makes it user-friendly for untrained users to carry out the complex and high throughput operations. The reusable plastic comb binding spines greatly reduce the cost of fabricating paper-based valves. To evaluate the performance, the actual samples of Fe (II) and nitrite were successfully analyzed. We hope this method will introduce a new approach to fabrication of paper-based valves on $\mu$ PADs in the future.
\end{abstract}

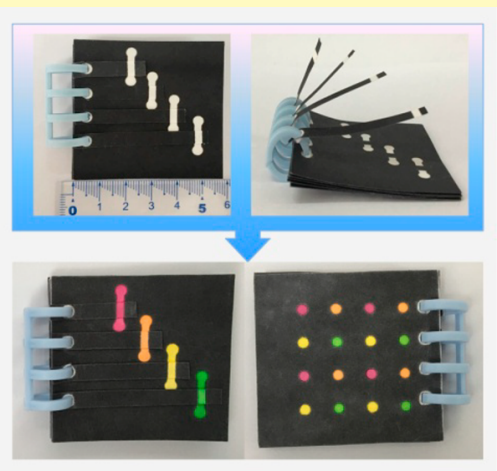

KEYWORDS: microfluidic paper-based analytical devices, paper-based valves, plastic comb binding spines, colorimetric detection, microfluidic chip, nitrite

$\mathrm{T}$ he paper-based microfluidic chip, as a branch of microfluidics, was first introduced in 2007 by Martinez et al. from Harvard University. ${ }^{1}$ With the rapid development of analytical microfluidic paper-based analytical devices ( $\mu$ PADs), it has gained global attention in recent years. ${ }^{2-5}$ Unlike conventional microfluidic chips made of crystalline silicon, glass, and polymers such as polydimethylsiloxane (PDMS) and poly(methyl methacrylate) (PMMA), paper-based microfluidic chip is based on the paper as a substrate material. Through various technical means, the paper is patterned to form designed hydrophilic and hydrophobic regions, and the relative chemical (or biochemical) reaction is performed in the corresponding region. ${ }^{6,7}$ Based on the paper's own capillary action and the combination with various detection means, different targets can be detected successfully. There were many different strategies applied on the paper-based microfluidic chips such as colorimetric detection, ${ }^{8-13}$ electrochemical detection, ${ }^{14-19}$ fluorescence, ${ }^{20-24}$ and surface enhanced Raman spectroscopy. ${ }^{25,26}$ It has the advantages of low cost, easy transport, simple operation, and good biocompatibility. $^{27-30}$ Moreover, it showed great application prospects including food safety, environmental monitoring, point-of-care testing, ${ }^{31,32}$ and diagnostic detection, ${ }^{33,34}$ particularly in limited-remote areas. ${ }^{4,11,35}$
The driving of the fluid on the paper chip mainly depends on its own capillary force, which does not need an extra pump system to drive the fluid that is feasible to the onsite analysis and detection. ${ }^{23,36,37}$ However, this characteristic of the paper itself poses a challenge to the controllable manipulation of the paper chip fluid. Moreover, when the chip performs the complicated or multistep assays, it is urgently needed for the fluid to be manipulated, and the paper-based valve plays a crucial and essential role in fluid manipulation of $\mu$ PADs. ${ }^{38}$

There were various ways to achieve the $\mu$ PADs valve's functionality by relying on different mechanisms to manipulate the capillary-driven fluid movement. They mainly include (i) designing geometries with different channel lengths, widths, and shapes; ${ }^{39,40}$ (ii) using chemicals to adjust the flow rate of the flow path; ${ }^{41,42}$ (iii) using physical methods to maintain or stop fluid flow. ${ }^{37,43,44}$ A variety of valve devices on $\mu \mathrm{PAD}$ have been developed recently. ${ }^{38,43}$ Xiao et al. presented a novel paper-based magnetic valve which can control the time and turn on or off liquid flow in $\mu$ PADs. ${ }^{45}$ Toley et al. developed tunable-delay shunt valves to implement sequential delivery

Received: June 19, 2018

Accepted: August 6, 2018

Published: August 6, 2018 
and utilized expandable polymers to mechanically connect or disconnect the fluidic pathway. ${ }^{36,37}$ Whiteside's group developed "pop-up" electrochemical paper-based analytical device, and the switch of the flow path in this device can be achieved by folding and unfolding. ${ }^{14}$

However, these methods suffered some limitations. Through the flow delay by a channel's geometry, it was hard to control the fluid. When using chemicals to change the flow rate, the chemical materials themselves could easily cause unnecessary interference. The very popular sponge-based valve had a long response time but only worked once, because the compressed sponge cannot be restored to its original state when it has encountered liquid. For rivet-based valves, the expanded rivet cannot be reused the next time. With increasing demand on paper chips such as performing multistep or complicated assays, it is crucially urgent to make a simple, flexible, reusable, fast-response-time, and low-cost paper-based valve.

In this paper, we present a novel strategy of fabricating a paper-based valve using plastic comb binding spines (PCBS). To the best of our knowledge, this is the first application of PCBS on microfluidic paper-based devices. The PCBS-based valves dramatically simplify valve fabrication processing because the paper-based device can be easily aligned by a dozen sheets of paper chip rather than sticking together layerby-layer. First, the PCBS-based valves avoid the nonspecific adsorption and cross contamination using adhesive tape. Second, this method can be employed for implementing multiple processing steps and assay detection. Third, the valve's response time is very fast for only several seconds for triggering. Finally, the PCBS-based valves dramatically simplify valve fabrication processing. Moreover, the plastic comb binding spines could be reusable in different $\mu$ PADs which greatly reduce the valve's cost. We hope that this technique will provide a new approach for the fabrication of paper-based valves in a robust, easy, and simple way on $\mu$ PADs in the future.

\section{EXPERIMENTAL SECTION}

Materials and Chemicals. Whatman filter paper (No. 1) was purchased from Hangzhou Xinhua Paper Limited Company (Shanghai, China). Different dyes, PCBS, and hole puncher were obtained from local stores. 1,10-Phenanthroline (Phen), hydroxylamine hydrochloride, ferrous sulfate $\left(\mathrm{FeSO}_{4}\right)$, sodium acetate trihydrate $(\mathrm{NaAc})$, glacial acetic acid, $\mathrm{H}_{3} \mathrm{PO}_{4}$, and $\mathrm{NaNO}_{2}$ were purchased from Sinopharm Chemical Reagent (China). $\mathrm{N}$-(1Naphthyl)-ethylenediamine dihydrochloride (NEDD) and $p$-aminobenzenesulfonic acid (PABS) were purchased from Aladdin Biochemical Technology Co., Ltd. (Shanghai, China). Deionized water (resistivity $\geq 18.20 \mathrm{M} \Omega$, Milli-Q Millipore) used for the preparation of aqueous solution was obtained from a Millipore water purification system. All chemicals used in the experiment were commercially available as analytical reagent and without further purification. The commercial $8560 \mathrm{DN}$ solid-wax printer (Fuji Xerox, Japan) was used to print the hydrophilic and hydrophobic areas on the paper chip. The baking equipment was purchased from Boxun industry and commerce company (Shanghai, China) and the signal intensity was obtained by the homemade paper-based colorimetric detecting device.

Design and Fabrication of the Microfluidic Paper-Based Chip. A designed paper chip was created by Illustrator drawing software and the hydrophilic and hydrophobic areas were printed on Whatman grade 1 by a XEROX Phaser 8560DN wax printer. For the wax to permeate through the paper, the printed drawings were placed in an oven at $150{ }^{\circ} \mathrm{C}$ for $30 \mathrm{~s}$.

Design and Fabrication of the Rotary Valve Paper-Based Device. A novel paper-based valve device was fabricated based on the paper chip designed above, which was similar to a desk calendar that can be free to page; the schematic diagram was showed in Figure 1.

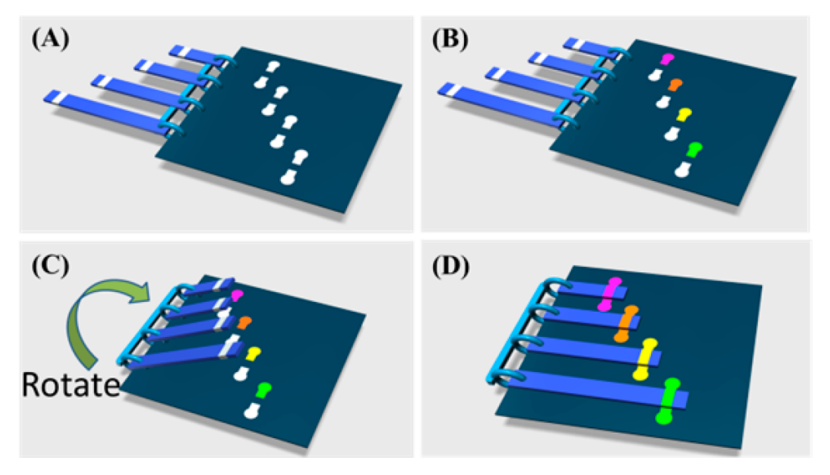

Figure 1. Schematic diagram of the paper-based valve device $(55 \mathrm{~mm}$ $\times 55 \mathrm{~mm}$ ) with PCBS. (A) The paper-based valve device opened the rotary channels ("OFF" State). (B) Dyes were added dropwise, and the channels were not connected when the rotary channels were opened. (C) Rotating the four rotary channels of the paper-based valve device. (D) The paper-based valve device closed the rotary channels ("ON" State), and dyes flowed in the channels.

The device was simple, facile, and portable. In addition, the cheap PCBS (Figure S1) was easily acquired from local stores. The length of every PCBS was $280 \mathrm{~mm}$, which can be cut into different lengths according to the needs of the experiments. For convenience to bind the paper-based chip, the length we cut out was $32 \mathrm{~mm}$, which contained four rings. The inner diameter of the ring was $11 \mathrm{~mm}$, and the outer diameter was $15 \mathrm{~mm}$. The side of the rings can be opened or closed, and the distance between each ring was $6 \mathrm{~mm}$, which determines the distance of the hole. Considering the actual situation, the diameter of the punching hole was $4.5 \mathrm{~mm}$, and the spacing of each hole was $5 \mathrm{~mm}$. It should be noted that the hole of each sheet of paper chip should be consistent to ensure the smooth flow of liquids. By controlling the paper layer flipping over and back, the valve's "ON/OFF" function can be realized to manipulate the fluid (Figure S2).

\section{RESULTS AND DISCUSSION}

Paper-Based Valve Based on PCBS for Fluid Detection. We designed a series of paper-based devices based on PCBS with different flow channels to test the performance of paper-based valves. The first paper-based device (approximately $55 \mathrm{~mm}$ wide $\times 55 \mathrm{~mm}$ long $\times 3 \mathrm{~mm}$ thick) was assembled with a nine-layered chip (Figure S3). The top layer was composed of four long-arm rotary channels, which were applied to control the connection or disconnection to the second layer's channel. Then layers of patterned paper were stacked in such a way that channels in adjacent layers of paper connect with each other. And finally, there were 64 test spots at the bottom layer, which could meet the needs of general testing and guarantee good repeatability. When any of the rotary channels in the first layer were closed, and the corresponding channels of the other layer were connected, then the sample could pass through the middle layers to reach the corresponding area at the bottom layer. For better visualization, different colored dyes (1:5 diluted in distilled water) were used for subsequent experiments. As illustrated in Figure 2, after the dyes were dropped in the sample area, if the rotary channels of the first layer were all opened ("OFF" State) (Figure $2 \mathrm{~A}, \mathrm{~A}^{\prime}$ ), the channels of the second layer cannot be connected, and there was no color observed on the bottom layer. In contrast, if the first rotary channel was closed ("ON" 


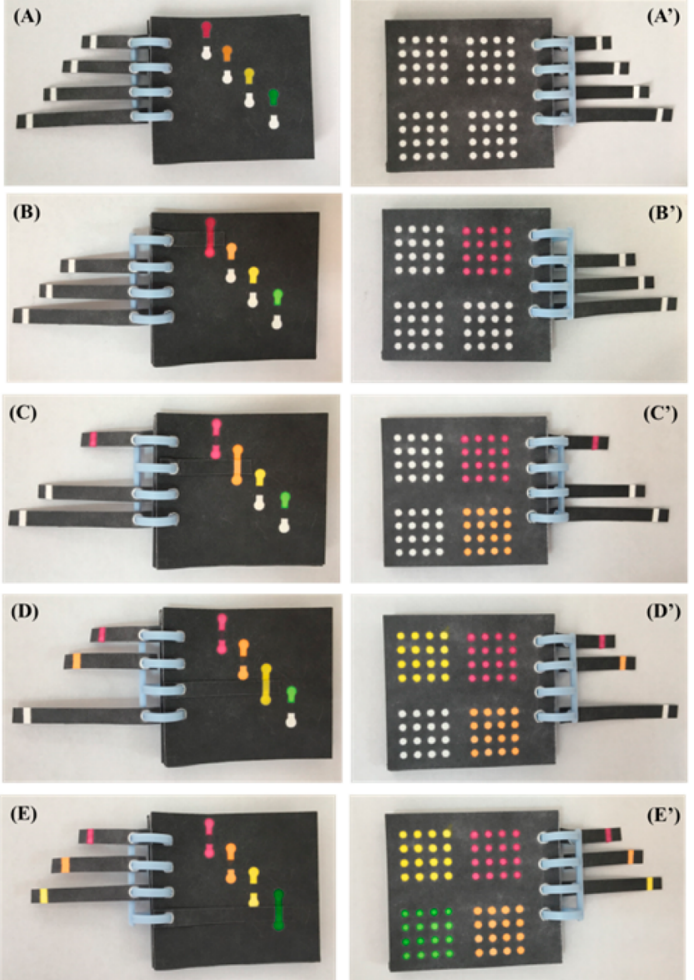

Figure 2. Front and back view images of paper-based valve device (55 $\mathrm{mm} \times 55 \mathrm{~mm}$ ) with opening or closing the rotary channels, when adding different dyes. $\left(A, A^{\prime}\right)$ The rotary channels of first layer were all opened, and there was no color on the bottom layer. (B-E) The first, second, third, and fourth rotary channels were closed sequentially; the pink, orange, yellow, and green dyes were diverted to corresponding channels, and $\left(\mathrm{B}^{\prime}-\mathrm{E}^{\prime}\right)$ showed the back of the paper-based valve devices that were distributed by the four dyes sequentially.

State) (Figure 2 B, B'), the pink dye could continuously be diverted to the sample area, and then the pink dye quickly spread to whole channels and the bottom layer showed only the pink dyes a few minutes later. In the same way, the second, third, and fourth rotary channels were closed ("ON" State) sequentially (Figure $2 \mathrm{C}-\mathrm{E}$ ), and then the orange, yellow, and green dyes could quickly be distributed to the corresponding detection regions on the bottom layer (Figure $2 \mathrm{C}^{\prime}-\mathrm{E}^{\prime}$ ). Notably, this kind of PCBS valve had a very fast response time (just several minutes) compared with the sponge-based valve, ${ }^{37,46}$ and could work many times by rotating. In addition, this valve can be designed conveniently with "ON" or "OFF" styles.

To further verify whether these PCBS valves could be applied to more complicated $\mu \mathrm{PADs}$, we designed two different paper-based devices to test the performance. The paper-based devices had 16 detection zones at the bottom, and can detect four samples simultaneously. As shown in Figure $3 \mathrm{~A}$, we designed 7 layers of paper chips (Figure 3C) and assembled them into a paper-based device using PCBS. During the experiment, first, all the rotary channels of the first paper chip were opened ("OFF" State), and four different-colored dyes (pink, orange, yellow, and green) were successively added to the sample injection area of the first layer in sequence. After waiting a few minutes, it was clearly observed that the bottommost paper chip had no color. Then, we closed the rotary channels ("ON" State) one by one to connect the channels,

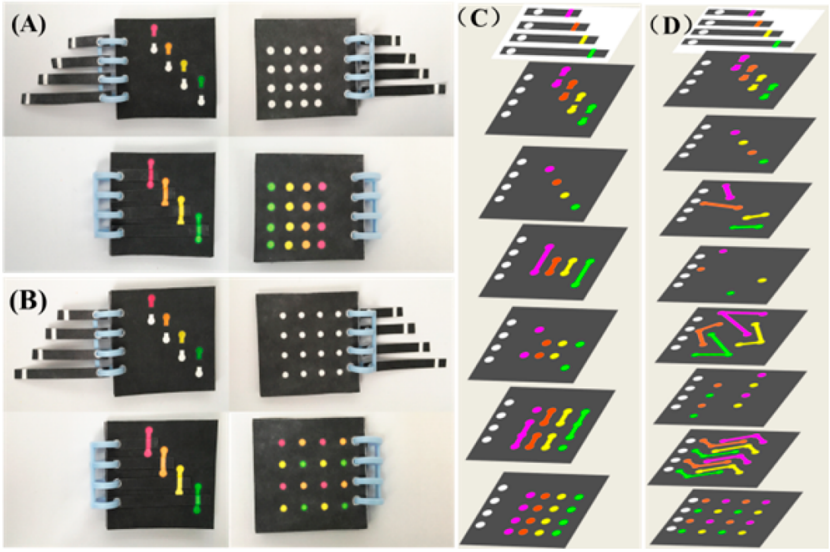

Figure 3. Design of different channel paper-based valve devices (55 $\mathrm{mm} \times 55 \mathrm{~mm}$ ) which were composed of 7 or 9 layers of paper chips that are distributed into four samples. $(\mathrm{A}, \mathrm{B})$ All the rotary channels of the first paper chip were opened ("OFF" State), and different dyes (pink, orange, yellow, and green) were added to the sample injection. There was no color on the bottom layer. The rotary channels of the first layer were all closed ("ON" State), and the locations of the 16 output regions were distributed in four columns or disorder, respectively. (C,D) Expanded views of two different paper-based valve devices.

and the bottom paper layer showed different colors in order. Another paper-based device was composed of 9 layers of paper chips (Figure 3D). In the same way, the bottom paper layer displayed different color distribution (Figure 3B). As a result, we can quickly and easily implement different detection requirements by designing relative flow channels based on PCBS-assisted valve.

Colorimetric Detection of Fe (II) and Nitrite. To test the usability of the paper-based PCBS valves, we measured the $\mathrm{Fe}$ (II) and nitrite on the paper-based device by the colorimetric method. The principle of detecting Fe (II) is that $\mathrm{Fe}$ (II) reacts with $o$-phenanthroline in the $\mathrm{pH}$ value of 39 to generate a stable orange complex, which requires that the $\mathrm{Fe}$ (III) reduced to $\mathrm{Fe}$ (II) by hydroxylamine hydrochloride before the reaction. According to the previous reports, ${ }^{47}$ an acetate buffer was prepared with $15.0 \mathrm{~g}$ of sodium acetate and $11.75 \mathrm{~mL}$ of glacial acetic acid in $50 \mathrm{~mL}$ of DI water, and the final concentration of acetate solution was $6.3 \mathrm{M}(\mathrm{pH} 4.5)$. The $8 \mathrm{mg} / \mathrm{mL}$ 1,10-phenanthroline and $0.1 \mathrm{mg} / \mathrm{mL}$ hydroxylamine were then prepared with the acetate buffer, respectively. On the paper-based devices of Figure 3A, $3 \mu \mathrm{L}$ hydroxylamine and $3 \mu \mathrm{L}$ 1,10-phenanthroline were added to the detection area of the bottom layer and air-dried in a few minutes, respectively. Then the rotary channel was closed and $3 \mu \mathrm{L} \mathrm{Fe}$ (II) sample with different concentrations (33, 67, 167, 333, $500,667 \mathrm{mg} / \mathrm{L}$ ) was added to the injection area. It is obvious that the color of the paper changed from white to orange within 2 min and the intensity curve is shown in Figure 4A; the intensity of the color was linearly dependent upon the amount of the $\mathrm{Fe}$ (II) with a linearity range $33.33-666.67 \mathrm{mg} / \mathrm{L}$. The LOD was calculated to $8.9 \mathrm{mg} / \mathrm{L}$. With increasing heavy metal pollution, this detection method can be used for on-site detection of other heavy metal ions, which is quick and convenient.

To further verify the usability of the PCBS paper-based device, we utilized nitrite as another test target in this device. Nitrite is a chemical pollutant that is widely present in the 

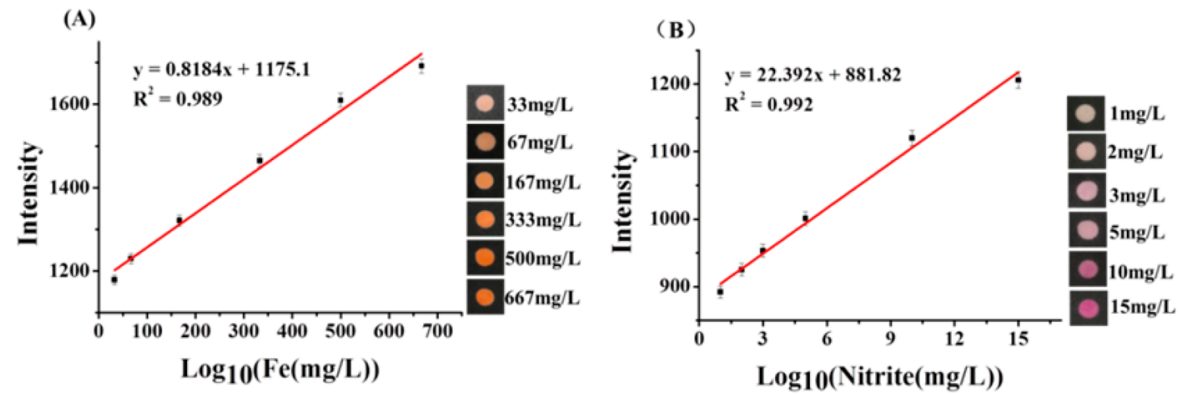

Figure 4. Calibration curves for determination of the target ions. (A) Calibration curve of different Fe (II) concentration (33, 67, 167, 333, 500, $667 \mathrm{mg} / \mathrm{L})$. (B) Calibration curve of different nitrite concentration $(1,2,3,5,10,15 \mathrm{mg} / \mathrm{L})(n=4)$.

Table 1. Recoveries of Fe (II) and $\mathrm{NO}_{2}{ }^{-}$in the Local Lake Water ${ }^{a}$

\begin{tabular}{|c|c|c|c|c|c|}
\hline \multirow[b]{2}{*}{ sample } & \multirow[b]{2}{*}{ added (mg/L) } & \multicolumn{2}{|c|}{ our method } & \multicolumn{2}{|c|}{ UV detection } \\
\hline & & detected $(\mathrm{mg} / \mathrm{L})$ & recovery $(\%)$ & $\operatorname{detected}(\mathrm{mg} / \mathrm{L})$ & recovery $(\%)$ \\
\hline \multirow[t]{3}{*}{$\mathrm{Fe}$ (II) } & 50 & $52.42 \pm 2.87$ & 104.8 & $51.5 \pm 3.34$ & 103.0 \\
\hline & 250 & $262.58 \pm 3.08$ & 105.3 & $256.75 \pm 3.74$ & 102.7 \\
\hline & 450 & $467.86 \pm 3.25$ & 103.9 & $459.03 \pm 5.38$ & 102.0 \\
\hline \multirow[t]{3}{*}{$\mathrm{NO}_{2}^{-}$} & 0.5 & $0.54 \pm 0.11$ & 108.0 & $0.53 \pm 0.13$ & 106.3 \\
\hline & 4.5 & $4.74 \pm 0.32$ & 105.3 & $4.69 \pm 0.17$ & 104.3 \\
\hline & 8.5 & $8.85 \pm 0.25$ & 104.1 & $8.76 \pm 0.23$ & 103.0 \\
\hline
\end{tabular}

${ }^{a}$ The standard deviation was obtained by four measurements in our paper-based devices and the comparison with the standard UV detection $(n=$ 4).

environment. It is closely related to people's diet and health. The principle of detecting nitrite is that nitrite diazotizes with $\mathrm{PABS}$ in $\mathrm{pH}<1.7$, and reacts in a coupling reaction with NEDD to generate a purple-red azo dye. According to the previous reports, ${ }^{8}$ the chromogenic reagent was prepared by a mixture of $50 \mathrm{~mL} \mathrm{H}_{3} \mathrm{PO}_{4}, 20 \mathrm{~g} \mathrm{PABS}$, and $1 \mathrm{~g}$ NEDD in DI water with the final volume of $500 \mathrm{~mL}$. On the paper-based device of Figure $3 \mathrm{~A}$, briefly, $3 \mu \mathrm{L}$ chromogenic reagent was added to the detection area of the bottom layer and air-dried in a few minutes, and then the rotary channel was connected and $3 \mu \mathrm{L}$ nitrite with different concentrations $(1,2,3,5,10,15$ $\mathrm{mg} / \mathrm{L}$ ) was introduced to the injection area and a color reaction held for $2 \mathrm{~min}$ for determination. As demonstrated in Figure 4B, the intensity of the color showed a linear correlation with the concentration of the nitrite; the dynamic linear range was determined from 1 to $15 \mathrm{mg} / \mathrm{L}$. The LOD was calculated to $0.28 \mathrm{mg} / \mathrm{L}$. The results demonstrated that our newly developed PCBS could be successfully used for the detection of Fe (II) and nitrite in environmental water samples.

Application of the PCBS Paper-Based Devices. To demonstrate the potential application of the PCBS paper-based device, the $\mathrm{Fe}$ (II) and $\mathrm{NO}_{2}{ }^{-}$in the local lake water were detected. Lake water samples were taken from Sanyuan lake of Yantai university (Yantai, China). The spiked samples were added, and the standard deviation was obtained by four measurements in our paper-based device and the comparison with the standard UV detection $(n=4)$. As shown in Table 1 , $\mathrm{Fe}$ (II) spiked concentrations were $50 \mathrm{mg} / \mathrm{L}, 250 \mathrm{mg} / \mathrm{L}$, and $450 \mathrm{mg} / \mathrm{L}$, the recoveries of the samples on the PCBS paperbased device were $104.8 \%, 103.9 \%$, and $105.3 \%$, respectively. From the table, we could observe that the detection results on the PCBS paper-based device were generally consistent with the results of the standard UV detection. The same conclusion can be drawn by detecting nitrite in lake samples, which indicated that the PCBS paper-based device had a good performance and could be used general testing.
In addition, we had discussed the theoretical understanding of liquid flow on the PCBS paper-based device. We designed a simple paper-based device (approximately $55 \mathrm{~mm}$ wide $\times 25$ $\mathrm{mm}$ long), which was assembled with only two layers with serpentine flow channels and actuated valve channel (Figure $5 \mathrm{~A})$, to test the flow speed of the paper-based valve. The width
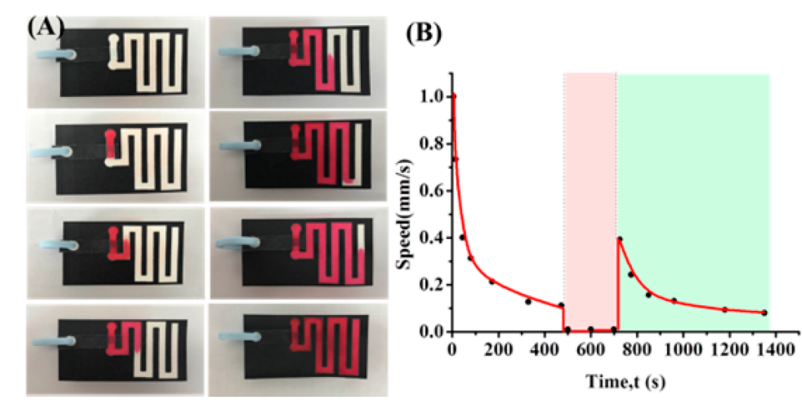

Figure 5. Time-lapse images of paper-based device with the serpentine channel actuated by PCBS valves. (A) Pictures of fluid traveling at different times. (B) Velocity curve of fluid migration. When closing the rotary channel at $0-472$ s ("ON" State), the speed of the fluid fell smoothly; when opening the rotary channel at 473709 s ("OFF" State), the speed of the fluid was 0; and when closing the rotary channel at 710-1350 s, the speed of the fluid fell gently and was obviously lower than the previous.

of channel was $3 \mathrm{~mm}$, the length was $171 \mathrm{~mm}$, and the red dye was added as a fluid to the channel. According to the classical Lucas-Washburn equation

$$
L^{2}=\frac{\gamma \cdot \cos \theta \cdot D}{2 \eta} \cdot t
$$

where $L$ means the length the fluid has penetrated the material, $\gamma$ is the fluid surface tension, $\cos \theta$ is the contact angle for the 
three-phase system (here, fluid, solid, air), $D$ represents the average pore diameter, and $\eta$ is the fluid viscosity. ${ }^{36,48}$

The whole process diagram was shown in Figure 5A. First, we closed the rotary channel to make the whole channel connected. When the red dye was introduced in a sample reservoir at the beginning, it gradually dyed the channel with a red color. With the time increasing, the liquid flow rate was gradually reduced (white region in Figure 5B), and this phenomenon can be explained by the Lucas-Washburn equation. The entire process was finished in $472 \mathrm{~s}$. When we opened the rotary channel, the flow rate decreased to zero (pink zone in Figure 5B). It implied that the PCBS valve had good function to control the fluid transportation. When the valve was closed again at $710 \mathrm{~s}$, the fluid front continued to migrate and dye the channel with a red color. It can be observed that the speed of the fluid then fell gently and was lower than the previous (green region in Figure 5B). The entire wicking process was finished at $1350 \mathrm{~s}$. Evaporation effects were controlled by enclosing devices in a closed room with constant humidity.

\section{CONCLUSIONS}

A novel strategy for fabricating the valves on a microfluidic paper-based analytical device was described and it can control fluid in a user-friendly way. The entire valve manipulation process was similar to a desk calendar that can be flipped over and turned back. The movable paper channel in a different layer could implement the channel's connection or disconnection to realize the valve function using plastic comb binding spines. The valve's response time only required several seconds. PCBS, as the important component of the paperbased valve, could be reusable many times, which greatly reduced the cost of fabricating the valves. This valve can move flexibly in space and this advantage enables it to be reusable with good repeatability. In addition, as a proof-of-concept, Fe (II) and nitrite have been investigated on this platform and showed good sensitivity and reproducibility. We hope this technique will provide a robust, easy, and simple approach for the fabrication of paper-based valves on $\mu$ PADs.

\section{ASSOCIATED CONTENT}

\section{(5) Supporting Information}

The Supporting Information is available free of charge on the ACS Publications website at DOI: 10.1021/acssensors.8b00518.

Pictures of PCBS, paper-based valve device and handheld device, expanded view of the paper-based valve device, and UV absorption spectra for $\mathrm{Fe}(\mathrm{II})$ and nitrite (PDF)

\section{AUTHOR INFORMATION}

\section{Corresponding Authors}

*E-mail: bwli@yic.ac.cn.

*E-mail: 1xchen@yic.ac.cn.

\section{ORCID}

Bowei Li: 0000-0001-6262-8248

Lingxin Chen: 0000-0002-3764-3515

\section{Notes}

The authors declare no competing financial interest.

\section{ACKNOWLEDGMENTS}

This work was financially supported by the National Natural Science Foundation of China (Grant No. 41776110), the Science and Technology Development Plan of Yantai (Grant No. 2015ZH087 and 2017ZH089), the National Key Research and Development Program of China (Grant No. 2016YFC1400702), Jilin Province's Science and Technology Department Project (Grant No. 20160204010SF), and the Project Sponsored by the Scientific Research Foundation for the Returned Overseas Chinese Scholars, State Education Ministry.

\section{REFERENCES}

(1) Martinez, A. W.; Phillips, S. T.; Butte, M. J.; Whitesides, G. M. Patterned Paper as a Platform for Inexpensive, Low-Volume, Portable Bioassays. Angew. Chem., Int. Ed. 2007, 46, 1318-1320.

(2) Martinez, A. W.; Phillips, S. T.; Whitesides, G. M. Three Dimensional Microfluidic Devices Fabricated in Layered Paper and Tape. Proc. Natl. Acad. Sci. U. S. A. 2008, 105, 19606-19611.

(3) Martinez, A. W.; Phillips, S. T.; Carrilho, E.; Thomas, S. W., III; Sindi, H.; Whitesides, G. M. Simple Telemedicine for Developing Regions: Camera Phones and Based Microfluidic Devices for RealTime, Off-Site Diagnosis. Anal. Chem. 2008, 80, 3699-3707.

(4) Yetisen, A. K.; Akram, M. S.; Lowe, C. R. Paper-Based Microfluidic Point-of-Care Diagnostic Devices. Lab Chip 2013, 13, 2210-2251.

(5) Li, B.; Zhang, Z.; Qi, J.; Zhou, N.; Qin, S.; Choo, J.; Chen, L. Quantum Dot-Based Molecularly Imprinted Polymers on ThreeDimensional Origami Paper Microfluidic Chip for Fluorescence Detection of Phycocyanin. ACS Sens. 2017, 2, 243-250.

(6) Carrilho, E.; Martinez, A. W.; Whitesides, G. M. Understanding Wax Printing: a Simple Micropatterning Process for Paper-Based Microfluidics. Anal. Chem. 2009, 81, 7091-7095.

(7) Lu, Y.; Shi, W.; Jiang, L.; Qin, J.; Lin, B. Rapid Prototyping of Paper-Based Microfluidics with Wax for Low-cost, Portable Bioassay. Electrophoresis 2009, 30, 1497-1500.

(8) Li, B.; Fu, L.; Zhang, W.; Feng, W.; Chen, L. Portable PaperBased Device for Quantitative Colorimetric Assays Relying on Light Reflectance Principle. Electrophoresis 2014, 35, 1152-1159.

(9) Sun, X.; Li, B.; Qi, A.; Tian, C.; Han, J.; Shi, Y.; Lin, B.; Chen, L. Improved Assessment of Accuracy and Performance Using a Rotational Paper-based Device for Multiplexed Detection of Heavy Metals. Talanta 2018, 178, 426-431.

(10) Gerold, C. T.; Bakker, E.; Henry, C. S. Selective Distance-Based $\mathrm{K}^{+}$Quantification on Paper-Based Microfluidics. Anal. Chem. 2018, 90, 4894-4900.

(11) Wang, H.; Li, Y.-j.; Wei, J.-f.; Xu, J.-r.; Wang, Y.-h.; Zheng, G.-x. Paper-Based Three-Dimensional Microfluidic Device for Monitoring of Heavy Metals with a Camera Cell Phone. Anal. Bioanal. Chem. 2014, 406, 2799-2807.

(12) Chan, H.; Shu, Y.; Xiong, B.; Chen, Y.; Chen, Y.; Tian, Q.; Michael, S.; Shen, B.; Wu, H. Simple, Cost-Effective 3D Printed Microfluidic Components for Disposable, Point-of-Care Colorimetric Analysis. ACS Sens. 2016, 1, 227-234.

(13) Srisa-Art, M.; Boehle, K. E.; Geiss, B. J.; Henry, C. S. Highly Sensitive Detection of Salmonella Typhimurium Using a Colorimetric Paper-Based Analytical Device Coupled with Immunomagnetic Separation. Anal. Chem. 2018, 90, 1035-1043.

(14) Wang, C.-C.; Hennek, J. W.; Ainla, A.; Kumar, A. A.; Lan, W.J.; Im, J.; Smith, B. S.; Zhao, M.; Whitesides, G. M. A Paper-Based "Pop-Up" Electrochemical Device for Analysis of Beta-Hydroxybutyrate. Anal. Chem. 2016, 88, 6326-6333.

(15) Sun, X.; Li, B.; Tian, C.; Yu, F.; Zhou, N.; Zhan, Y.; Chen, L. Rotational Paper-Based Electrochemiluminescence Immunodevices for Sensitive and Multiplexed Detection of Cancer Biomarkers. Anal. Chim. Acta 2018, 1007, 33-39. 
(16) Li, W. P.; Li, L.; Li, S.; Wang, X.; Li, M.; Wang, S. W.; Yu, J. H. 3D Origami Electrochemiluminescence Immunodevice Based on Porous Silver-Paper Electrode and Nanoporous Silver DoubleAssisted Signal Amplification. Sens. Actuators, B 2013, 188, 417-424.

(17) Ding, J.; Li, B.; Chen, L.; Qin, W. A Three-Dimensional Origami Paper-Based Device for Potentionmetric Biosensing. Angew. Chem., Int. Ed. 2016, 55, 13033-13037.

(18) Zhang, Y.; Li, L.; Zhang, L.; Ge, S.; Yan, M.; Yu, J. In-Situ Synthesized Polypyrrole-cellulose Conductive Networks for PotentialTunable Foldable Power Paper. Nano Energy 2017, 31, 174-182.

(19) Cuartero, M.; Crespo, G. A.; Bakker, E. Paper-Based ThinLayer Coulometric Sensor for Halide Determination. Anal. Chem. 2015, 87, 1981-90.

(20) Qi, J.; Li, B.; Wang, X.; Zhang, Z.; Wang, Z.; Han, J.; Chen, L. Three-Dimensional Paper-Based Microfluidic Chip Device for Multiplexed Fluorescence Detection of $\mathrm{Cu}^{2+}$ and $\mathrm{Hg}^{2+}$ Ions Based on Ion Imprinting Technology. Sens. Actuators, B 2017, 251, 224233.

(21) Noor, M. O.; Shahmuradyan, A.; Krull, U. J. Paper-Based SolidPhase Nucleic Acid Hybridization Assay Using Immobilized Quantum Dots as Donors in Fluorescence Resonance Transfer. Anal. Chem. 2013, 85, 1860-1867.

(22) Ju, Q.; Noor, M. O.; Krull, U. J. Paper-Based Biodetection Using Luminescent Nanoparticles. Analyst 2016, 141, 2838-2860.

(23) Choi, J. R.; Liu, Z.; Hu, J.; Tang, R.; Gong, Y.; Feng, S.; Ren, H.; Wen, T.; Yang, H.; Qu, Z.; Pingguan-Murphy, B.; Xu, F. Polydimethylsiloxane-Paper Hybrid Lateral Flow Assay for Highly Sensitive Point-of-Care Nucleic Acid Testing. Anal. Chem. 2016, 88, 6254-64.

(24) Caglayan, M. G.; Sheykhi, S.; Mosca, L.; Anzenbacher, P. Fluorescent Zinc and Copper Complexes for Detection of Adrafinil in Paper-Based Microfluidic Devices. Chem. Commun. 2016, 52, 827982.

(25) Li, B.; Zhang, W.; Chen, L.; Lin, B. A Fast and Low-Cost Spray Method for Prototyping and Depositing Surface-Enhanced Raman Scattering Arrays on Microfluidic Paper Based Device. Electrophoresis 2013, 34, 2162-2168.

(26) Zhang, W.; Li, B.; Chen, L.; Wang, Y.; Gao, D.; Ma, X.; Wu, A. Brushing, a Simple Way to Fabricate SERS Active Paper SubStrates. Anal. Methods 2014, 6, 2066-2071.

(27) Liu, W.; Cassano, C. L.; Xu, X.; Fan, Z. H. Laminated PaperBased Analytical Devices (LPAD) with Origami-Enabled Chemiluminescence Immunoassay for Cotinine Detection in Mouse Serum. Anal. Chem. 2013, 85, 10270-10276.

(28) Ge, L.; Wang, S.; Song, X.; Ge, S.; Yu, J. 3D Origami-Based Multifunction-Integrated Immunodevice: Low-Cost and Multiplexed Sandwich Chemiluminescence Immunoassay on Microfluidic PaperBased Analytical Device. Lab Chip 2012, 12, 3150-3158.

(29) Tian, T.; Li, J.; Song, Y.; Zhou, L.; Zhu, Z.; Yang, C. J. Distance-Based Microfluidic Quantitative Detection Methods for Point-of-Care Testing. Lab Chip 2016, 16, 1139-1151.

(30) Sanjay, S. T.; Fu, G.; Dou, M.; Xu, F.; Liu, R.; Qi, H.; Li, X. Biomarker Detection for Disease Diagnosis Using Cost-Effective Microfluidic Platforms. Analyst 2015, 140, 7062-7081.

(31) Li, Z.; Li, F.; Xing, Y.; Liu, Z.; You, M.; Li, Y.; Wen, T.; Qu, Z.; Li, X. L.; Xu, F. Pen-on-Paper Strategy for Point-of-Care Testing: Rapid Prototyping of Fully Written Microfluidic Biosensor. Biosens. Bioelectron. 2017, 98, 478-485.

(32) Renault, C.; Li, X.; Fosdick, S. E.; Crooks, R. M. HollowChannel Paper Analytical Devices. Anal. Chem. 2013, 85, 7976-9.

(33) Hu, J.; Wang, S.; Wang, L.; Li, F.; Pingguan-Murphy, B.; Lu, T. J.; Xu, F. Advances in Paper-Based Point-of-Care Diagnostics. Biosens. Bioelectron. 2014, 54, 585-97.

(34) Liu, Z.; Hu, J.; Zhao, Y.; Qu, Z.; Xu, F. Experimental and Numerical Studies on Liquid Wicking into Filter Papers for PaperBased Diagnostics. Appl. Therm. Eng. 2015, 88, 280-287.

(35) Meredith, N. A.; Quinn, C.; Cate, D. M.; Reilly, T. H.; Volckens, J., III; Henry, C. S. Paper-Based Analytical Devices for Environmental Analysis. Analyst 2016, 141, 1874-1887.
(36) Toley, B. J.; McKenzie, B.; Liang, T.; Buser, J. R.; Yager, P.; Fu, E. Tunable-Delay Shunts for Paper Microfluidic Devices. Anal. Chem. 2013, 85, 11545-11552.

(37) Toley, B. J.; Wang, J. A.; Gupta, M.; Buser, J. R.; Lafleur, L. K.; Lutz, B. R.; Fu, E.; Yager, P. A Versatile Valving Toolkit for Automating Fluidic Operations in Paper Microfluidic Devices. Lab Chip 2015, 15, 1432-1444.

(38) Fu, E.; Downs, C. Progress in the Development and Integration of Fluid Flow Control Tools in Paper microfluidics. Lab Chip 2017, $17,614-628$.

(39) Giokas, D. L.; Tsogas, G. Z.; Vlessidis, A. G. Programming Fluid Transport in Paper-Based Microfluidic Devices Razor-Crafted Open Channels. Anal. Chem. 2014, 86, 6202-6207.

(40) Noh, N.; Phillips, S. T. Metering the Capillary-Driven Flow of Fluids in Paper-Based Microfluidic Devices. Anal. Chem. 2010, 82, 4181-4187.

(41) Lutz, B.; Liang, T.; Fu, E.; Ramachandran, S.; Kauffman, P.; Yager, P. Dissolvable Fluidic Time Delays For Programming MultiStep Assays in Instrument-Free Paper Diagnostics. Lab Chip 2013, 13, 2840-2847.

(42) Wei, X.; Tian, T.; Jia, S.; Zhu, Z.; Ma, Y.; Sun, J.; Lin, Z.; Yang, C. J. Target-Responsive DNA Hydrogel Mediated "Stop-Flow" Microfluidic Paper-Based Analytic Device for Rapid, Portable and Visual Detection of Multiple Targets. Anal. Chem. 2015, 87, 42754282.

(43) Li, B.; Yu, L.; Qi, J.; Fu, L.; Zhang, P.; Chen, L. Controlling Capillary-Driven Fluid Transport in Paper-Based Microfluidic Devices Using a Movable Valve. Anal. Chem. 2017, 89, 5707-5712.

(44) Liu, H.; Crooks, R. M. Three-Dimensional Paper Microfluidic Devices Assembled Using the Principles of Origami. J. Am. Chem. Soc. 2011, 133, 17564-17566.

(45) Li, X.; Zwanenburg, P.; Liu, X. Magnetic Timing Valves for Fluid Control in Paper-Based Microfluidics. Lab Chip 2013, 13, 2609-2614.

(46) Fu, E.; Liang, T.; Spicar-Mihalic, P.; Houghtaling, J.; Ramachandran, S.; Yager, P. Two-Dimensional Paper Network Format That Enables Simple Multistep Assays for Use in LowResource Settings in the Context of Malaria Antigen Detection. Anal. Chem. 2012, 84, 4574-9.

(47) Mentele, M. M.; Cunningham, J.; Koehler, K.; Volckens, J.; Henry, C. S. Microfluidic Paper-Based Analytical Device for Particulate Metals. Anal. Chem. 2012, 84, 4474-80.

(48) Fu, E.; Lutz, B.; Kauffman, P.; Yager, P. Controlled Reagent Transport in Disposable 2D Paper Networks. Lab Chip 2010, 10, 918-20. 